\title{
A seismometer phase-sensitive detection circuit design
}

\author{
Xuefeng Xing ${ }^{1,2, a^{*}, \text { Huan Liu }}{ }^{1}$,Shixue Lv', Guanyu Zhang ${ }^{1}$, Yun Long ${ }^{1}$ and \\ Junqiu Wang ${ }^{1}$
}

${ }^{1}$ College of Instrumentation and Electrical Engineering, Jilin University, Changchun, P. R. China

${ }^{2}$ National Instrument Engineering Technology Research Center of geophysical exploration, Jilin University, Changchun, P. R. China

${ }^{a}$ xing@jlu.edu.cn

Keywords: Capacitance sensor. Phase sensitive demodulation. Operational amplifier.

Abstract. Phase-sensitive detection circuit tests signals according to the correlation of the reference signal and the input signal. The noise is eliminated by the independence between the reference signal and the noise signal. The sensitivity of the detector is improved significantly by the suppression of the noise. In this work, a phase-sensitive detection circuit is designed for seismometer, which possesses the characteristics of low-input noise, variable-output gain and high sensitivity. The feasibility of this phase-sensitive detection circuit is verified by the Multisim and the signal-to-noise ratio is satisfactory.

\section{Introduction}

With the development of society, the prediction and the early warning of the earthquake are attracting more and more attention, the requirement of the precise seismic geophone is also increased ${ }^{[1-8]}$. Seismic geophones are the instruments used to convert the signals of the earth shake into computer-readable digital images/signals for analysis. The major components of the seismic geophone included transducer, drive circuit, detection circuit, communication circuit and data storage unit. In the traditional geophone, transducer, drive circuit and detection circuit are the all components, the recorder takes the place of communication circuit and data storage unit. In the application of conventional earth exploration, $0 \sim 300 \mathrm{~Hz}$ is the general frequency range of the effective perception, and in the reflection seismic exploration, the signals from low frequency and high frequency are low-energy. Therefore, there is increasing interest in developing detection instruments to further improve detection performance.

In the low frequency vibration detection, a high requirement of the detection circuit is necessary since the intensity of the signal is comparable with the intensity of the noise. Capacitance displacement sensor have many advantages, such as well temperature stability, simple structure, strong adaptability, good dynamic response, high accuracy and low-cost, etc. Recently, the development of the integrated circuit technology is rapid and it has become the most widely used detection technology. In this case, capacitance displacement sensor plays a major role in the various measurement fields because of the high-sensitivity and high-precision. Phase-sensitive detection circuit is the key circuit of the integrated circuit technology, and is the most important part of the detection system.

In this work, a phase-sensitive detection circuit is designed for seismometer, which possesses the characteristics of low-input noise, variable-output gain and high sensitivity. The feasibility of this 
phase-sensitive detection circuit is verified by the Multisim and the signal-to-noise ratio is satisfactory.

\section{Experiment principle}

Phase-sensitive detection circuit tests signals according to the correlation of the reference signal and the input signal. The noise is eliminated by the independence between the reference signal and the noise signal. The sensitivity of the detector is improved significantly by the suppression of the noise. The experiment principle of phase-sensitive detector was shown in Figure 1.

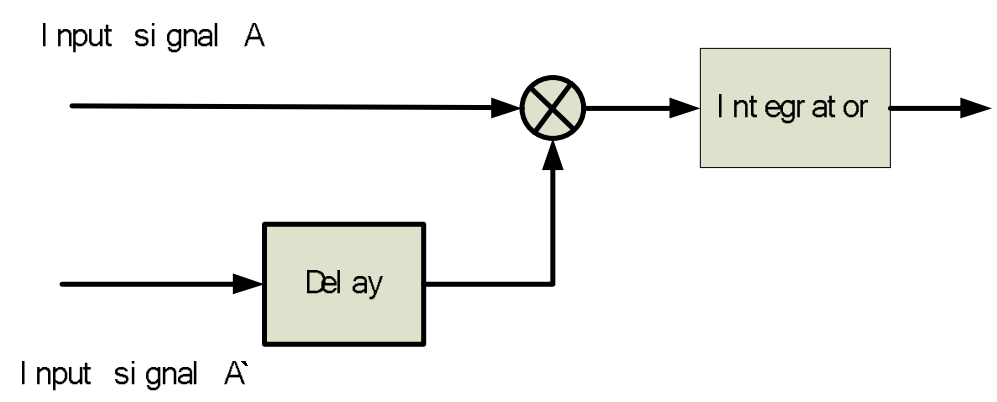

Figure 1. Phase-sensitive detection principle diagram.

Phase-sensitive detector achieves multiplication in phase-sensitive demodulation. The process of the operation is through the integration of the low-pass filter to complete between input signal and reference signal cross-correlation operation, the operation will be measured capacitance signal into a dc voltage signal. Commonly used phase-sensitive demodulation has multiplication phase-sensitive demodulation and switch phase-sensitive demodulation, the principles of these demodulations were shown in Figure 2.
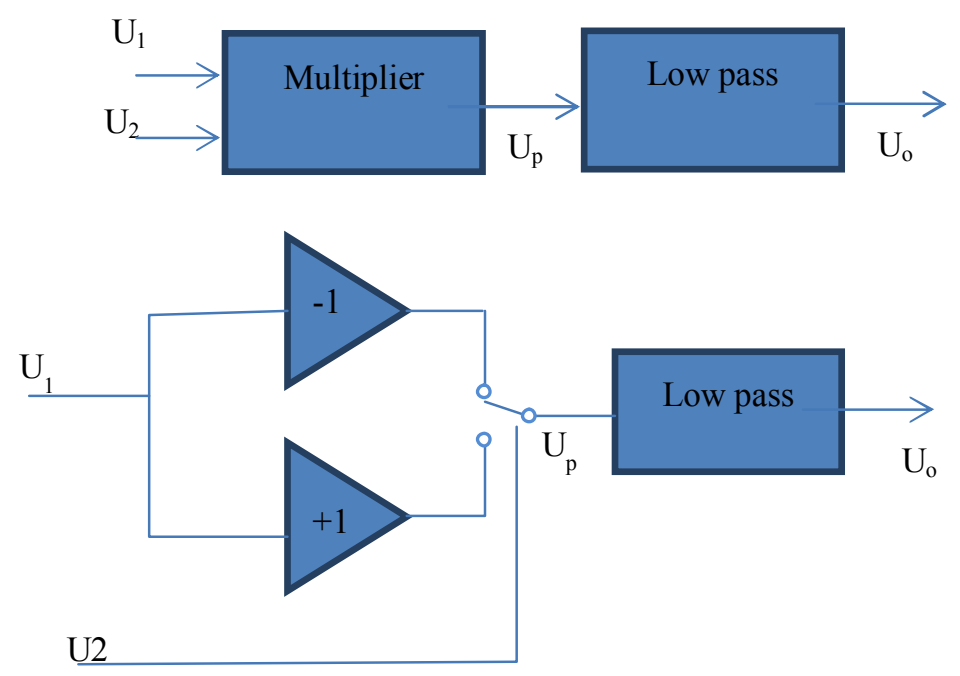

Figure 2 The diagram of multiplication phase-sensitive demodulation and switch phase-sensitive demodulation. 


\section{Circuit design}

The comparator phase-sensitive detection scheme was used in this system. With the main design features including the use of filter and the high sensitivity operational amplifier, the comparator phase-sensitive detection scheme is simple structure and low parts count. The circuit design of the phase-sensitive detector was shown in Figure 3.

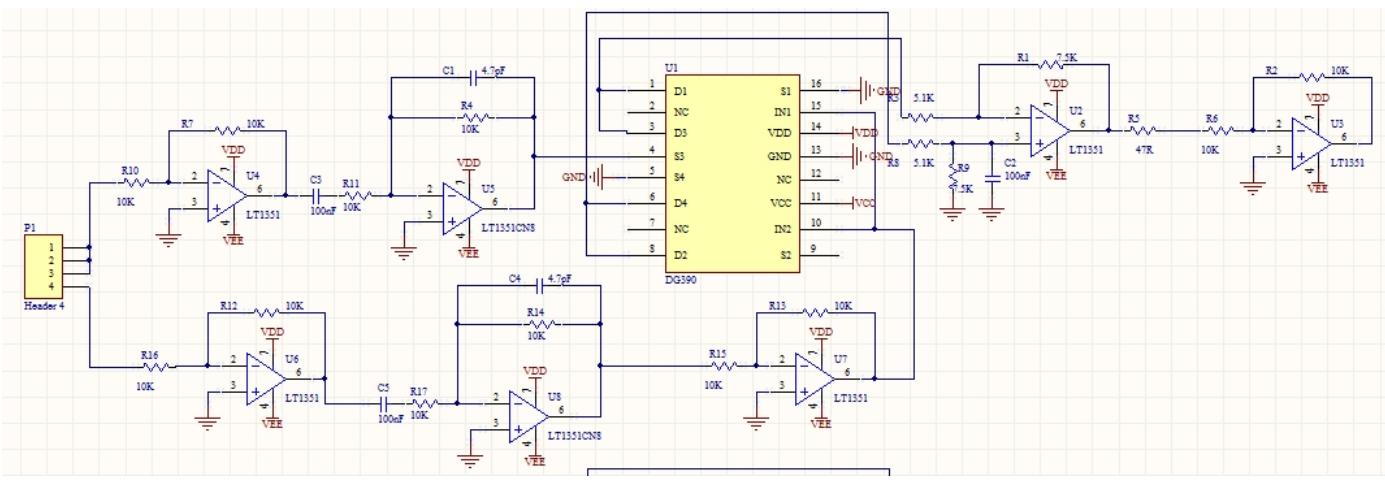

Figure 3. Phase-sensitive detector circuit diagram.

\section{Circuit simulation results}

Circuit simulation results using Multisim was shown in Figure 4. The results indicated that phase sensitive wave circuit output positive and negative dc component, the circuit was stability and the noise was controlled at $15 \mathrm{nV} / \sqrt{H z}$ level.
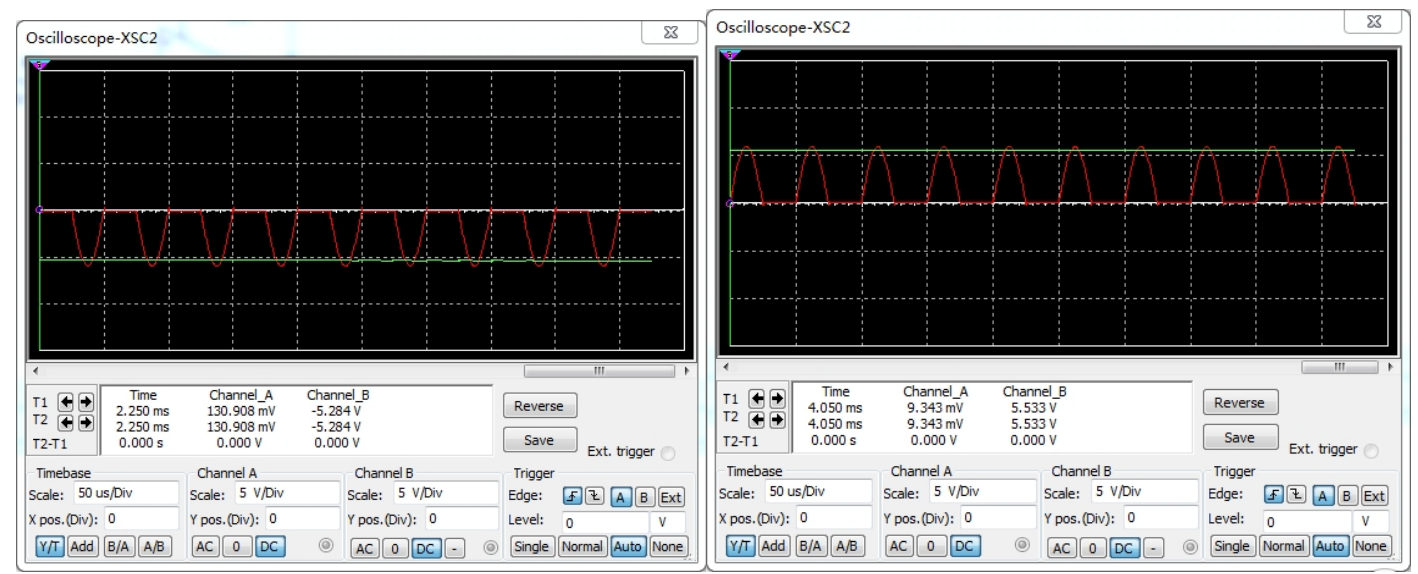

Figure 4. The circuit simulations of the phase-sensitive detector.

\section{Conclusion}

A phase-sensitive detection circuit was developed for seismic geophone by the comparison method with low switching devices count after analyzing the existing phase-sensitive detection circuit. The feasibility of the circuit and the stability of the long- operating time were verified by simulation. There is no obvious fluctuation and drift. The problem of the inconsistent of voltage between the negative output and the positive output will be studied and discussed in the further research. 


\section{References}

[1] T. Dean, Dupuis J.C., and Hassan R. The coherency of ambient seismic noise recorded during land surveys and the resulting implications for the effectiveness of geophone arrays, Geophysics, 2015, 80, (3), pp. P1-P10

[2] A.G. Tsakiris, Papanicolaou A.N., and Lauth T.J. Signature of bedload particle transport mode in the acoustic signal of a geophone, J Hydraul Res, 2014, 52, (2), pp. 185-204

[3] S.H. Joh, Abd Rahman N., Ramli B., and Han K.H. Alleviation of numerical instability in geophone-based measurement of track vibrations by utilizing a priori information, Ksce J Civ Eng, 2014, 18, (5), pp. 1351-1358

[4] B. Fahrman, Westman E., Karfakis M., and Luxbacher K. Optimization of Geophone Array for Monitoring Geologic Carbon Sequestration Using Double-Difference Tomography, J Energ Resour-Asme, 2014, 136, (1)

[5] D.E. Voigt, Peters L.E., and Anandakrishnan S. 'Georods': the development of a four-element geophone for improved seismic imaging of glaciers and ice sheets, Ann Glaciol, 2013, 54, (64), pp. 142-148

[6] Z.H. Sun, Zhang F.X., Li S.J., Wang C., Peng G.D., Li H.G., and Wu X.B. Field test of a DFB fiber laser geophone system, 2013 International Conference on Optical Instruments and Technology: Optical Sensors and Applications, 2013, 9044

[7] S.E. Dosso Three-dimensional Acoustic Localization in the Arctic using an Ice-mounted Geophone, Oceans-Ieee, 2013

[8] M. Zubair, Hartmann K., and Loffeld O. Design and Implementation of a Wireless Geophone Sensor Node for Target Detection and Classification, Automatic Target Recognition Xxii, 2012, 8391 